\title{
Hydrostatically pressurized buckling of complete spherical shells filled with an elastic medium
}

\author{
Motohiro SATO*, M. Ahmer WADEE**, Takafumi SEKIZAWA***, \\ Kohtaroh IIBOSHI**** and Hiroyuki SHIMA ${ }^{\dagger}$ \\ *Member Dr.,Eng. Assistant Prof. Div. Engineering and Policy for Sustainable Environment, \\ Hokkaido Univ. (Kita 13, Nishi 8, Kita-ku, Sapporo) and \\ Visiting Lecturer, Dept. Civil and Environmental Engineering, South Kensington Campus, \\ Imperial College London (London SW7 2AZ, UK) \\ ${ }^{* *}$ Non-Member PhD Reader in Nonlinear Mechanics, Dept. Civil and Environmental Engineering, \\ South Kensington Campus, Imperial College London (London SW7 2AZ, UK) \\ *** Member Undergraduate Student. Div. Engineering and Policy for Sustainable Environment, \\ Hokkaido Univ. (Kita 13, Nishi 8, Kita-ku, Sapporo) \\ ${ }^{* * * *}$ Member Graduate Student. Div. Engineering and Policy for Sustainable Environment, \\ Hokkaido Univ. (Kita 13, Nishi 8, Kita-ku, Sapporo) \\ ${ }^{\dagger}$ Non-Member Assistant Prof. Div. Applied Physics, Hokkaido Univ.
}

(Kita 13, Nishi 8, Kita-ku, Sapporo)

The critical buckling characteristics of hydrostatically pressurized complete spherical shells filled with an elastic medium are presented. A model based on small deflection thin shell theory, the equations of which are solved using exact methods in conjunction with variational principles, is presented. In the current formulation, axisymmetric and inextensional assumptions are not used initially and the elastic medium is modelled as a Winkler foundation, i.e. using uncoupled radial springs with a constant foundation modulus that is independent of wave numbers of shell buckling modes. Critical buckling pressures and characteristic modal shapes are demonstrated for a wide range of material and geometric parameters. A phase diagram is established to obtain the requisite thickness to radius, and stiffness ratios for a desired mode profile. The present formulation can be readily extended to apply to more general cases of non-axisymmetric buckling problems.

Key Words : hydrostatically pressurized buckling, complete spherical shell, Winkler foundation

\section{Introduction}

The analytical study of the structural behaviour of spherical shells is of great importance in the fields of not only civil, mechanical and aeronautical engineering but also nanoscience and biomechanics. Notable examples include pressure vessels, spherical honeycombs $^{1)}$, carbon onions ${ }^{2)}$ and spherical viruses ${ }^{3)}$ and so on. In the more conventional engineering research fields, some pioneering theoretical works on the elastic instability issues of empty complete spherical shells were conducted in 1960 s by Thompson ${ }^{4)}$, Hutchinson 5) and Koiter ${ }^{6)}$.

In recent years, analytical works which considered an interaction between a spherical shell and an internal elastic medium have also been conducted. The interaction leads to novel buckling patterns which depend on the stiffness and thickness to radius ra- tios. Recently for example, Yin et al. ${ }^{7)}$ successfully demonstrated the stress-driven buckling patterns in spheroidal core/shell structures, where the core implies an elastic medium, by using the finite element approach. Interestingly, the authors insisted in that work ${ }^{7}$ ) that shapes of many natural fruits and vegetables can be reproduced by anisotropic stressdriven buckles on the spheroidal core/shell system (see Fig. 1).

Purely from a structural mechanics perspective, Timoshenko ${ }^{8)}$, and Flügge ${ }^{9}$ ) introduced the formulation for buckling of a complete spherical shell in their respective books that are now regarded as classics in the field. In these, the exact approaches to solve the buckling problem of hydrostatically pressurized complete spherical shells without an internal elastic medium were introduced. However, both of these formulations were based on the axisymmetric 


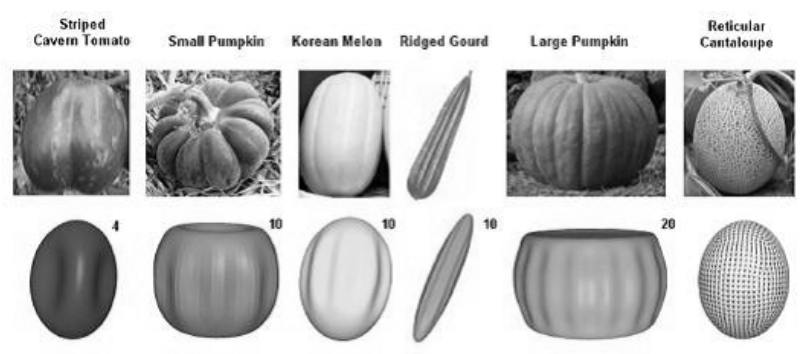

Fig. 1 Comparison between several fruits (or vegetables) and examples of corresponding simulated buckle shapes on model spheroids (reprinted with permission from Yin et al. $\left.(2008)^{7)}\right)$.

assumption and formulations for more general cases including non-axisymmetric deformations were not included. More recently, Fok and Allwrite ${ }^{10)}$ analysed the elastic axisymmetric buckling behaviour of a complete spherical shell embedded in an elastic material and loaded by a far-field hydrostatic pressure. In that study, the energy method in conjunction with a Rayleigh-Ritz trial function was used for simplicity but the validation of the obtained results was not discussed in detail with buckling deformation modes being omitted. Hence, to our knowledge, no general nonaxisymmetric formulation, in conjunction with exact methods of solution, has been developed for the buckling behaviour of complete spherical shells with an internal elastic medium thus far. Moreover, much attention has been given to the structural morphology of the core/shell structure. Some authors have recently demonstrated the cross-sectional morphology of carbon nanotubes embedded in an elastic medium ${ }^{11), 12)}$. These results clearly show that interactions between shells and cores lead to some novel wavy-shaped buckling deformation patterns.

From the background described above, the buckling properties of hydrostatically pressurized complete spherical shells filled with an elastic medium are demonstrated in the current study. Presently, the elastic medium is modelled as a Winkler foundation, i.e. with uncoupled radial springs and a constant foundation modulus. An exact approach for the solution of the developed equations based on the formulation without using the axisymmetric and inextensional assumptions is presented. This approach therefore avoids any discussion about the validity of the solution and allows to model to be extended to cover more generic non-axisymmetric cases with relative ease. The analytical results are presented with a phase diagram and illustrative buckling modes that are compared with earlier results. Conclusions are then drawn.

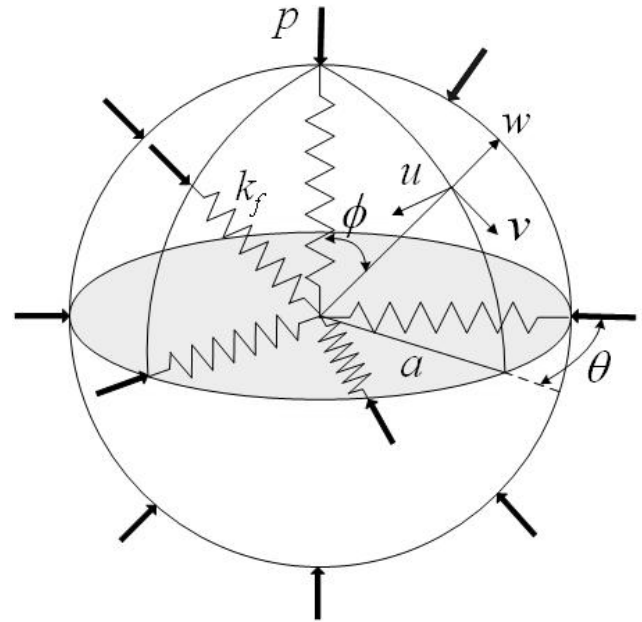

Fig. 2 Hydrostatically pressurized spherical shell filled with an elastic medium.

\section{Analytical model}

Figure 2 shows the analytical model considered in the current study. The critical pitchfork bifurcation phenomenon of the hydrostatically pressurized complete spherical shell is investigated. The spherical shell is constructed from an homogeneous and isotropic linear elastic material with Young's modulus $E$ and Poisson's ratio $\nu$, which is filled with an elastic material that is modelled as a Winkler foundation, i.e. with uncoupled springs in the radial direction with a constant foundation modulus $k_{f}$. For a complete spherical shell with radius $a$ and thickness $h$, spherical angular coordinates in the latitude and meridian directions $\theta$ and $\phi$ are used respectively. Displacement functions: $u, v$ and $w$ are in the $\theta, \phi$ and the (outward) radial directions, respectively.

\section{Formulation}

\subsection{Energy formulation}

The following analysis is based on classical small deformation theory of thin shells 13),14). The total potential energy $V$ is expressed by the sum of the strain energy and work done by external load as:

$$
V=U_{M}+U_{B}+U_{F}+\Omega
$$

in which $U_{M}$ is the membrane (in-plane) strain energy term, where:

$$
\begin{aligned}
& U_{M}=U_{M \phi}+U_{M \theta}+U_{M \phi \theta}, \\
& U_{M \phi}=\frac{1}{2} \int_{\phi} \int_{\theta} N_{\phi} \epsilon_{\phi} a^{2} \sin \phi \mathrm{d} \phi \mathrm{d} \theta, \\
& U_{M \theta}=\frac{1}{2} \int_{\phi} \int_{\theta} N_{\theta} \epsilon_{\theta} a^{2} \sin \phi \mathrm{d} \phi \mathrm{d} \theta, \\
& U_{M \phi \theta}=\int_{\phi} \int_{\theta} N_{\phi \theta} \epsilon_{\phi \theta} a^{2} \sin \phi \mathrm{d} \phi \mathrm{d} \theta,
\end{aligned}
$$


$U_{B}$ is the bending (out-of-plane) component, where:

$$
\begin{aligned}
& U_{B}=U_{B \phi}+U_{B \theta}+U_{B \phi \theta}, \\
& U_{B \phi}=\frac{1}{2} \int_{\phi} \int_{\theta} M_{\phi} \chi_{\phi} a^{2} \sin \phi \mathrm{d} \phi \mathrm{d} \theta, \\
& U_{B \theta}=\frac{1}{2} \int_{\phi} \int_{\theta} M_{\theta} \chi_{\theta} a^{2} \sin \phi \mathrm{d} \phi \mathrm{d} \theta, \\
& U_{B \phi \theta}=\int_{\phi} \int_{\theta} M_{\phi \theta} \chi_{\phi \theta} a^{2} \sin \phi \mathrm{d} \phi \mathrm{d} \theta,
\end{aligned}
$$

$U_{F}$ is the strain energy term due to a Winkler foundation, thus:

$$
U_{F}=\frac{1}{2} \int_{\phi} \int_{\theta} a^{2} k_{f} w^{2} \sin \phi \mathrm{d} \phi \mathrm{d} \theta,
$$

and $\Omega$ is the potential energy of the applied hydrostatic pressure $p$ :

$$
\Omega=\int_{\phi} \int_{\theta} a^{2} p w \sin \phi \mathrm{d} \phi \mathrm{d} \theta
$$

Based on the assumptions of thin shell theory ${ }^{13)}$, the strain-displacement relations can be expressed as:

$$
\begin{aligned}
\epsilon_{\phi} & =\frac{1}{a}\left(v,_{\phi}+w\right), \\
\epsilon_{\theta} & =\frac{1}{a}\left(\cot \phi v+\frac{u,_{\theta}}{\sin \phi}+w\right), \\
\epsilon_{\phi \theta} & =\frac{1}{a}\left(\frac{v,_{\theta}}{\sin \phi}-\cot \phi u+u,_{\phi}\right), \\
\beta_{\phi} & =\frac{1}{a}\left(-w,_{\phi}+v\right), \\
\beta_{\theta} & =\frac{1}{a}\left(-\frac{w,_{\theta}}{\sin \phi}+u\right), \\
\chi_{\phi} & =\frac{1}{a^{2}}\left(-w,_{\phi \phi}+v,_{\phi}\right), \\
\chi_{\theta} & =\frac{1}{a^{2}}\left[-\frac{w,_{\theta \theta}}{\sin ^{2} \phi}+u,,_{\theta}+\cot \phi\left(v-w,_{\phi}\right)\right], \\
\chi_{\phi \theta} & =\frac{\sin \phi u,_{\phi}+v,_{\theta}-2 w,,_{\phi \theta}+2 \cot \phi w,_{\theta}-\cos \phi u}{2 a^{2} \sin \phi},
\end{aligned}
$$

where ", $x$ " denotes differentiation with respect to $x$. The constitutive relationships are as follows:

$$
\begin{aligned}
& N_{\phi}=C\left(\epsilon_{\phi}+\nu \epsilon_{\theta}\right), \quad N_{\theta}=C\left(\epsilon_{\theta}+\nu \epsilon_{\phi}\right), \\
& N_{\phi \theta}=C \frac{1-\nu}{2} \epsilon_{\phi \theta}, \quad M_{\phi}=D\left(\chi_{\phi}+\nu \chi_{\theta}\right), \\
& M_{\theta}=D\left(\chi_{\theta}+\nu \chi_{\phi}\right), \quad M_{\phi \theta}=D \frac{1-\nu}{2} \chi_{\phi \theta},
\end{aligned}
$$

where $C=E h /\left(1-\nu^{2}\right)$ is the membrane stiffness and $D=E h^{3} / 12\left(1-\nu^{2}\right)$ is the flexural rigidity of the spherical shell, respectively. Substituting the expressions in Eq.(6) and (7) into Eqs.(2)-(4) gives the energy expressions in terms of the displacements $u, v$ and $w$.

\subsection{Fundamental state}

When uniform hydrostatic pressure $p$ is acting on the spherical shell, only the inward radial static displacement $w_{0}$ can occur in the pre-buckling state. Hence, the stress-strain relationship of the spherical shell is assumed to be linear up to the point of instability. In the fundamental pre-buckling state, the potential energies $V^{(0)}$ are given by:

$V^{(0)}=U_{M}^{(0)}+U_{B}^{(0)}+U_{F}^{(0)}+\Omega^{(0)}=\int_{\phi} \int_{\theta} F^{(0)} \mathrm{d} \phi \mathrm{d} \theta$,

where:

$$
\begin{aligned}
U_{M}^{(0)} & =C(1+\nu) \int_{\phi} \int_{\theta} w_{0}^{2} \mathrm{~d} \phi \mathrm{d} \theta, \quad \mathrm{U}_{\mathrm{B}}^{(0)}=0, \\
U_{F}^{(0)} & =\frac{1}{2} \int_{\phi} \int_{\theta} a^{2} k_{f} w^{2} \sin \phi \mathrm{d} \phi \mathrm{d} \theta, \\
\Omega^{(0)} & =\int_{\phi} \int_{\theta} a^{2} p w_{0} \sin \phi \mathrm{d} \phi \mathrm{d} \theta .
\end{aligned}
$$

For an equilibrium state, the first variation of the total potential energy $V$ must equal zero. This condition gives the following static displacement under uniform hydrostatic pressure:

$$
w_{0}=-\frac{a^{2}(1-\nu)}{2 E h+a^{2} k_{f}(1-\nu)} p .
$$

\subsection{Critical buckling analysis}

\section{(1) Minimum potential energy criterion}

To obtain an expression for the second variation of the total potential energy, the following infinitesimally small increments are defined:

$$
u=u_{1}, \quad v=v_{1}, \quad w=w_{0}+w_{1},
$$

which correspond to buckling displacement modes. The expression for the potential energy due to the external force is a linear functional of the displacement components and makes no contribution to the second variation expression, i.e. $\delta^{2} \Omega=0{ }^{13)}$. Consequently, the second variation of the total potential energy becomes:

$$
\delta^{2} U=\delta^{2} U_{M}+\delta^{2} U_{B}+\delta^{2} U_{F}=\int_{\phi} \int_{\theta} F \mathrm{~d} \phi \mathrm{d} \theta
$$

where:

$$
\begin{aligned}
\delta^{2} U_{M}= & \delta^{2} U_{\text {stretch }}+\delta^{2} U_{\text {shear }}, \\
\delta^{2} U_{\text {stretch }}= & \frac{C w_{0}(1+\nu)}{a} \int_{\phi} \int_{\theta}\left[\left(v_{1}+w_{1}, \phi\right)^{2}\right. \\
& \left.+\left(u_{1}-w_{1},{ }_{\theta} \csc \phi\right)^{2}\right] \sin \phi \mathrm{d} \phi \mathrm{d} \theta \\
& +C \int_{\phi} \int_{\theta}\left[\left(v_{1}+w_{1},{ }_{\phi}\right)^{2}\right. \\
& \left.+\left(u_{1}-w_{1}, \theta \csc \phi\right)^{2}\right] \mathrm{d} \phi \mathrm{d} \theta, \\
\delta^{2} U_{\text {shear }}= & \frac{C(1-\nu)}{2} \int_{\phi} \int_{\theta}\left(\cot \phi u_{1}+\csc \phi v_{1}, \theta\right. \\
& \left.+u_{1},{ }_{\phi}\right)^{2} \sin \phi \mathrm{d} \phi \mathrm{d} \theta,
\end{aligned}
$$




$$
\begin{aligned}
\delta^{2} U_{B}= & \delta^{2} U_{\text {bend }}+\delta^{2} U_{\text {twist }} \\
\delta^{2} U_{\text {bend }}= & \frac{D}{a^{2}} \int_{\phi} \int_{\theta}\left\{\left(\cot \phi u_{1}+\csc \phi v_{1}, \theta\right.\right. \\
& \left.-\csc ^{2} \phi w_{1}, \theta \theta-\cot \phi w_{1}, \phi\right)^{2} \\
& \left(v_{1,{ }_{\phi}+w_{1}, \phi \phi}\right)\left[v_{1},{ }_{\phi}+w_{1},{ }_{\phi \phi}+\right. \\
& 2 \nu\left(\cot \phi v_{1}+\csc \phi u_{1}, \theta-\csc ^{2} \phi w_{1}, \theta \theta\right. \\
& \left.\left.\left.-\cot \phi w_{1},{ }_{\phi}\right)\right]\right\} \sin \phi \mathrm{d} \phi \mathrm{d} \theta \\
\delta^{2} U_{t w i s t}= & \frac{D(1-\nu)}{2 a^{2}} \int_{\phi} \int_{\theta}\left[2(1+\nu) w_{1}\right. \\
& \left(v_{1} \cot \phi+w_{1}+u_{1}, \theta+u_{1}, \phi\right) \\
& +u_{1, \phi}\left(2 \nu v_{1} \cot \phi+2 \nu u_{1, \theta}+v_{1, \phi}\right) \\
& \left.+\left(v_{1} \cot \phi+u_{1}, \theta \csc \phi\right)^{2}\right] \sin \phi \mathrm{d} \phi \mathrm{d} \theta \\
\delta^{2} U_{F} & =\int_{\phi} \int_{\theta} a^{2} k_{f} w_{1}^{2} \sin \phi \mathrm{d} \phi \mathrm{d} \theta
\end{aligned}
$$

According to the Trefftz criterion, the buckling equations can be obtained by introducing $F$ in Eq.(12) into the Euler-Lagrange equations with the calculus of variations. The Euler-Lagrange equations in this case are as follows ${ }^{13)}$ :

$$
\begin{gathered}
\frac{\partial F}{\partial u_{1}}-\frac{\partial}{\partial \phi}\left(\frac{\partial F}{\partial u_{1, \phi}}\right)-\frac{\partial}{\partial \theta}\left(\frac{\partial F}{\partial u_{1, \theta}}\right)=0, \\
\frac{\partial F}{\partial v_{1}}-\frac{\partial}{\partial \phi}\left(\frac{\partial F}{\partial v_{1, \phi}}\right)-\frac{\partial}{\partial \theta}\left(\frac{\partial F}{\partial v_{1, \theta}}\right)=0, \\
\frac{\partial F}{\partial w_{1}}-\frac{\partial}{\partial \phi}\left(\frac{\partial F}{\partial w_{1, \phi}}\right)-\frac{\partial}{\partial \theta}\left(\frac{\partial F}{\partial w_{1, \theta}}\right) \\
+\frac{\partial^{2}}{\partial \phi^{2}}\left(\frac{\partial^{2} F}{\partial w_{1, \phi \phi}}\right)+\frac{\partial^{2}}{\partial \phi \partial \theta}\left(\frac{\partial^{2} F}{\partial w_{1, \phi \theta}}\right) \\
+\frac{\partial^{2}}{\partial \theta^{2}}\left(\frac{\partial^{2} F}{\partial w_{1, \theta \theta}}\right)=0 .
\end{gathered}
$$

\section{(2) Stability equation}

Substitution of a solution of the form:

$$
\begin{aligned}
u_{1} & =\sum \bar{u}(\phi) \sin n \theta, \\
v_{1} & =\sum \bar{v}(\phi) \cos n \theta, \\
w_{1} & =\sum \bar{w}(\phi) \cos n \theta,
\end{aligned}
$$

where $n$ is a positive integer, into Eqs.(12) - (15) gives the following ordinary differential equations for arbitrary $n$ after some manipulation:

$$
\begin{gathered}
a_{u}^{(i)} \frac{\partial^{i} \bar{u}}{\partial \phi^{i}}+b_{u}^{(i)} \frac{\partial^{i} \bar{v}}{\partial \phi^{i}}+c_{u}^{(i)} \frac{\partial^{i} \bar{w}}{\partial \phi^{i}} \\
-\frac{w_{0}}{a}(1+\nu)(\bar{u} \sin \phi+n \bar{w})=0, \\
a_{v}^{(i)} \frac{\partial^{i} \bar{u}}{\partial \phi^{i}}+b_{v}^{(i)} \frac{\partial^{i} \bar{v}}{\partial \phi^{i}}+c_{v}^{(i)} \frac{\partial^{i} \bar{w}}{\partial \phi^{i}} \\
-\frac{w_{0}}{a}(1+\nu) \sin \phi\left(\bar{v}-\frac{\partial \bar{w}}{\partial \phi}\right)=0,
\end{gathered}
$$

$$
\begin{gathered}
a_{w}^{(i)} \frac{\partial^{i} \bar{u}}{\partial \phi^{i}}+b_{w}^{(i)} \frac{\partial^{i} \bar{v}}{\partial \phi^{i}}+c_{w}^{(i)} \frac{\partial^{i} \bar{w}}{\partial \phi^{i}}+\frac{a^{2} k_{f}}{C} \bar{w} \sin \phi \\
+\frac{w_{0}}{a}(1+\nu) \sin \phi\left(\frac{\partial \bar{v}}{\partial \phi}+\bar{v} \cot \phi+\frac{n \bar{u}}{\sin \phi}\right. \\
\left.-\frac{\partial^{2} \bar{w}}{\partial \phi^{2}}-\frac{\partial \bar{w}}{\partial \phi} \cot \phi+\frac{n^{2}}{\sin ^{2} \phi} \bar{w}\right)=0
\end{gathered}
$$

in which the implied summation over $i$ covers the range of non-zero coefficients, which are described, including the non-dimensional quantity $k=h^{2} / 12 a^{2}$, as follows:

$a_{u}^{(0)}=(1+k)\left[\frac{1-\nu}{2}\left(1-\cot ^{2} \phi\right) \sin \phi-\frac{n^{2}}{\sin \phi}\right]$,

$a_{u}^{(1)}=(1+k) \frac{1-\nu}{2} \cos \phi, a_{u}^{(2)}=(1+k) \frac{1-\nu}{2} \sin \phi$,

$a_{u}^{(3)}=a_{u}^{(4)}=0$,

$b_{u}^{(0)}=(1+k) \frac{\nu-3}{2} n \cot \phi, b_{u}^{(1)}=-(1+k) \frac{1+\nu}{2} n$,

$b_{u}^{(2)}=b_{u}^{(3)}=b_{u}^{(4)}=0$,

$c_{u}^{(0)}=k n\left(2-\frac{n^{2}}{\sin ^{2} \phi}\right)$

$c_{u}^{(1)}=k n \cot \phi, c_{u}^{(2)}=k n$,

$c_{u}^{(3)}=c_{u}^{(4)}=0$,

$a_{v}^{(0)}=(1+k) \frac{\nu-3}{2} n \cot \phi, a_{v}^{(1)}=(1+k) \frac{1+\nu}{2} n$,

$a_{v}^{(2)}=a_{v}^{(3)}=a_{v}^{(4)}=0$,

$b_{v}^{(0)}=-\frac{1+k}{\sin \phi}\left(\cos ^{2} \phi+\nu \sin ^{2} \phi+\frac{1-\nu}{2} n^{2}\right)$,

$b_{v}^{(1)}=(1+k) \cos \phi, b_{v}^{(2)}=(1+k) \sin \phi$,

$b_{v}^{(3)}=b_{v}^{(4)}=0$,

$c_{v}^{(0)}=-2 k n^{2} \frac{\cot \phi}{\sin \phi}, c_{v}^{(1)}=-k\left(2-\frac{1+n^{2}}{\sin ^{2} \phi}\right) \sin \phi$,

$c_{v}^{(2)}=-k \cos \phi, c_{v}^{(3)}=-k \sin \phi, c_{v}^{(4)}=0$,

$a_{w}^{(0)}=(1+k)(1+\nu) n-k n\left(2+\frac{1-n^{2}}{\sin ^{2} \phi}\right)$,

$a_{w}^{(1)}=k n \cot \phi, a_{w}^{(2)}=-k n, a_{w}^{(3)}=a_{w}^{(4)}=0$,

$b_{w}^{(0)}=(1+k)(1+\nu) \cos \phi-k \cos \phi\left(2+\frac{1-n^{2}}{\sin ^{2} \phi}\right)$,

$b_{w}^{(1)}=(1+k)(1+\nu) \sin \phi+\frac{k\left(n^{2}+\cos ^{2} \phi\right)}{\sin \phi}$,

$b_{w}^{(2)}=-2 k \cos \phi, b_{w}^{(3)}=-k \sin \phi, b_{w}^{(4)}=0$,

$c_{w}^{(0)}=-k\left[2(1+\nu) \sin \phi+n^{2} \frac{3-\nu+4 \cot ^{2} \phi}{\sin \phi}\right.$

$\left.-\frac{n^{4}}{\sin ^{3} \phi}\right], c_{w}^{(1)}=\left(1-\nu+\frac{1+2 n^{2}}{\sin ^{2} \phi}\right) \cos \phi$,

$c_{w}^{(2)}=-\frac{1+2 n^{2}+\nu \sin ^{2} \phi}{\sin \phi}$,

$c_{w}^{(3)}=2 k \cos \phi, c_{w}^{(4)}=k \sin \phi$.

These governing differential equations (18)-(20) are difficult to solve exactly by only assuming periodic 
functions for $\bar{u}, \bar{v}$ and $\bar{w}$ directly. However, the implementation of the following special linear differential operator enables the solution of these equations with an exact approach:

$$
H(\ldots)=(\ldots)^{\prime \prime}+(\ldots)^{\prime} \cot \phi+(\ldots)\left(2-\frac{n^{2}}{\sin ^{2} \phi}\right),
$$

where primes denote differentiation with respect to $\phi$. From the definition of $H$ in Eq.(22), the following relations are readily obtained:

$$
\begin{aligned}
H H(\ldots) & =(\ldots)^{\prime \prime \prime \prime}+2(\ldots)^{\prime \prime \prime} \cot \phi \\
& +(\ldots)^{\prime \prime}\left(3-\frac{1+2 n^{2}}{\sin ^{2} \phi}\right) \\
& +(\ldots)^{\prime} \cot \phi\left(4+\frac{1+2 n^{2}}{\sin ^{2} \phi}\right) \\
& +(\ldots)\left(4-\frac{2 n^{2}}{\sin ^{2} \phi}-\frac{n^{2}\left(4-n^{2}\right)}{\sin ^{4} \phi}\right), \\
H^{\prime}(\ldots)= & (\ldots)^{\prime \prime \prime}+(\ldots)^{\prime \prime} \cot \phi \\
& +(\ldots)^{\prime} \cot \phi\left(2-\frac{1+n^{2}}{\sin ^{2} \phi}\right) \\
& +2 n^{2}(\ldots) \frac{\cos \phi}{\sin ^{3} \phi} .
\end{aligned}
$$

In addition, a new variable $\hat{u}$ is defined in terms of $\bar{u}$ by:

$$
\bar{u}=\hat{u}^{\prime}
$$

By making use of the relations of Eqs.(22)-(24) and the new definition of Eq.(25), Eqs.(18) and (19) can be rewritten respectively as:

$$
\begin{aligned}
& (1+k) \sin \phi \frac{\partial}{\partial \phi}\left(\frac{1-\nu}{2} H(\hat{u})-\frac{1+\nu}{2} n \frac{\bar{v} \sin \phi+n \hat{u}}{\sin ^{2} \phi}\right) \\
& -2(1+k) n \frac{\bar{v} \sin \phi+n \hat{u}}{\sin ^{2} \phi} \cos \phi \\
& -(1+k)(1+\nu) n w+k n H(w) \\
& -\frac{w_{0}(1+\nu)}{a}\left(\hat{u}^{\prime}+n \bar{w}\right)=0, \\
& (1+k)\left[\frac{1+\nu}{2}(\bar{v} \sin \phi+n \hat{u})^{\prime \prime}+\frac{1-\nu}{2} H(\bar{v} \sin \phi)\right. \\
& \left.\quad-\frac{3-\nu}{2} \cot \phi(\bar{v} \sin \phi+n \hat{u})^{\prime}+(1+\nu) w^{\prime} \sin \phi\right] \\
& -k \sin \phi H^{\prime}(w)-\frac{w_{0}(1+\nu)}{a} \sin \phi\left(\bar{v}-\bar{w}^{\prime}\right)=0 .
\end{aligned}
$$

Differentiation of Eq.(27) with respect to $\phi$ and then multiplying it by $\sin \phi / n$ leads to the following:

$$
\begin{aligned}
& (1+k) \frac{\sin \phi}{n}\left\{\frac{1+\nu}{2}(\bar{v} \sin \phi+n \hat{u})^{\prime \prime \prime}\right. \\
& +\frac{1-\nu}{2} H^{\prime}(\bar{v} \sin \phi)+\frac{3-\nu}{2}\left[\frac{1}{\sin ^{2} \phi}(\bar{v} \sin \phi+n \hat{u})^{\prime}\right.
\end{aligned}
$$

$$
\begin{aligned}
& \left.-\cot \phi(\bar{v} \sin \phi+n \hat{u})^{\prime \prime}\right] \\
& \left.+(1+\nu)\left(w^{\prime \prime} \sin \phi+w^{\prime} \cos \phi\right)\right\} \\
& -k \frac{\sin \phi}{n}\left[\cos \phi H^{\prime}(w)+\sin \phi H^{\prime \prime}(w)\right]=0 .
\end{aligned}
$$

By adding Eq.(28) to Eq.(26), some algebraic manipulation gives:

$$
\begin{aligned}
& (1+k)[H(\Psi)-(1+\nu)(\Psi-H(w)+2 w)] \\
& \quad-k(H H(w)-2 H(w))-\frac{w_{0}}{a}(\Psi-H(w)+2 w)=0,
\end{aligned}
$$

in which:

$$
\Psi=\frac{(\bar{v} \sin \phi+n \hat{u})^{\prime}}{\sin \phi} .
$$

Similarly, Eq.(20) can be written using the operator $H$ and the new variable $\Psi$, defined in Eq.(30), as follows:

$$
\begin{aligned}
& (1+k)(1+\nu)(\Psi+2 w) \\
& \quad+k[H H(w)-(3+\nu) H(w)-H(\Psi)] \\
& \quad+\frac{a^{2} k_{F}}{C} w+(1+\nu) \frac{w_{0}}{a}(\Psi-H(w)+2 w)=0 .
\end{aligned}
$$

Equations (29) and (31) are the governing equations to be solved. Now, the solutions of $\Psi$ and $w$ of Eq.(29) and (31) are assumed to be in the form:

$$
\Psi=\sum_{m=0}^{\infty} A_{m} P_{m}(\cos \phi), \quad w=\sum_{m=0}^{\infty} B_{m} P_{m}(\cos \phi),
$$

where $A_{m}$ and $B_{m}$ are the constant deformation amplitudes, and the spherical harmonic $P_{m}(\cos \phi)$ is a series of Legendre functions of degree $n$ which satisfies the following Legendre differential equation:

$$
P_{m}^{\prime \prime}+P_{m}^{\prime} \cot \phi+m(m+1) P_{m}=0 .
$$

By using the operator $H$, defined in Eq.(22), the following relation can be obtained:

$$
H\left(P_{m}\right)=-\lambda_{m} P_{m}
$$

where:

$$
\lambda_{m}=m(m+1)-2,
$$

and

$$
H H\left(P_{m}\right)=-\lambda_{m}^{2} P_{m} .
$$

Substituting the series given in Eq.(32) into Eqs.(29) and (31) and using Eqs.(34) and (36), we obtain:

$$
\begin{aligned}
& \sum_{m=0}^{\infty}\left(c_{m}^{(11)} A_{m}+c_{m}^{(12)} B_{m}\right) P_{m}(\cos \phi)=0, \\
& \sum_{m=0}^{\infty}\left(c_{m}^{(21)} A_{m}+c_{m}^{(22)} B_{m}\right) P_{m}(\cos \phi)=0
\end{aligned}
$$




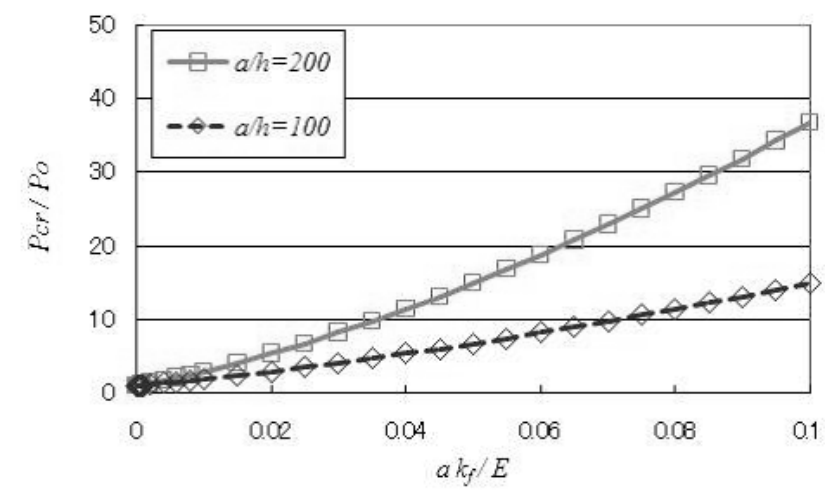

Fig. 3 Variation of the nondimensional critical buckling pressure $p_{c r} / p_{0}$ with $a k_{f} / E$.

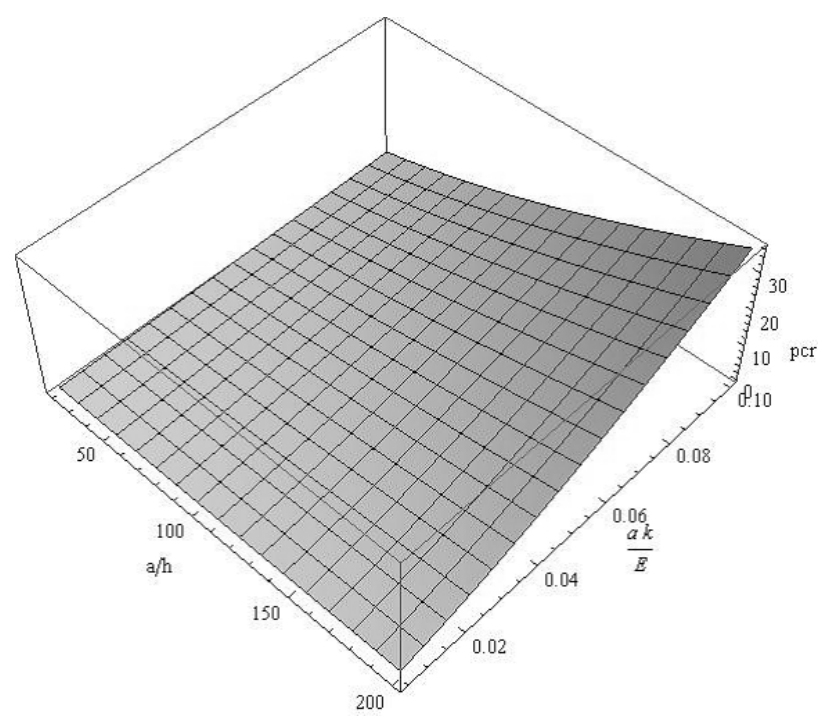

Fig. 4 Nondimensional critical pressure curves with $a k_{f} / E$ and $a / h$.

in which:

$$
\begin{aligned}
c_{m}^{(11)}= & (1+k)\left(\lambda_{m}+1+\nu\right)+\frac{w_{0}}{a}(1+\nu), \\
c_{m}^{(12)}= & (1+k)(1+\nu)+k\left(\lambda_{m}^{2}+2 k \lambda_{m}\right) \\
& +\frac{w_{0}}{a}(1+\nu)\left(1+\lambda_{m}\right), \\
c_{m}^{(21)}= & (1+k)(1+\nu)+k \lambda_{m}+\frac{w_{0}}{a}(1+\nu), \\
c_{m}^{(22)}= & 2(1+k)(1+\nu)+k \lambda_{m}\left(\lambda_{m}+3+\nu\right) \\
& +\frac{a^{2} k_{F}}{C}+\frac{w_{0}}{a}(1+\nu)\left(\lambda_{m}+2\right) .
\end{aligned}
$$

Hence, homogeneous linear equations for $A_{m}$ and $B_{m}$ are obtained for all $m$ as follows:

$$
\left[\begin{array}{ll}
c_{m}^{(11)} & c_{m}^{(12)} \\
c_{m}^{(21)} & c_{m}^{(22)}
\end{array}\right]\left(\begin{array}{l}
A_{m} \\
B_{m}
\end{array}\right)=\left(\begin{array}{l}
0 \\
0
\end{array}\right) .
$$

This is a standard eigenvalue problem for which nontrivial values are obtained when the coefficient matrix in Eq.(39) becomes singular. The corresponding

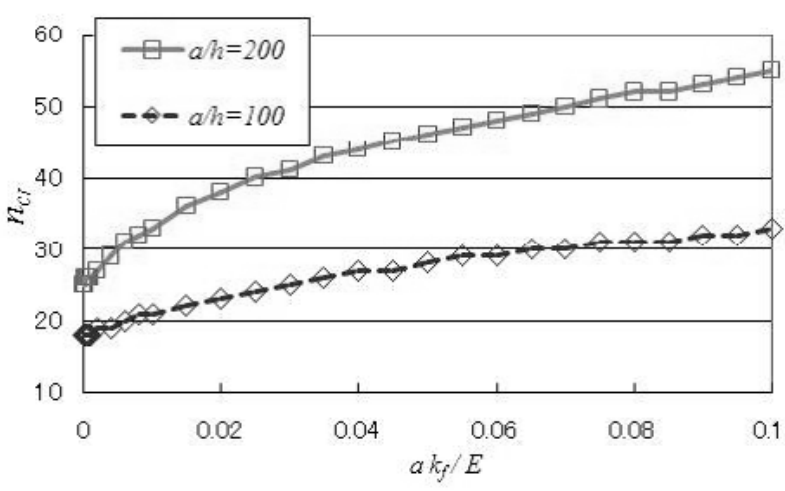

Fig. 5 Variation of the nondimensional critical buckling mode number $m_{c r}$.

pressure $p$ is thus calculated from the determinantal equations:

$$
\operatorname{det}\left[\begin{array}{cc}
c_{m}^{(11)} & c_{m}^{(12)} \\
c_{m}^{(21)} & c_{m}^{(22)}
\end{array}\right]=0
$$

By solving Eq.(40), the pressure $p$ with the corresponding mode $m$ for several combinations of geometric and material constants can be obtained as an eigenvalue. For the particular values of the constants, the minimum value of the eigenvalues is the critical buckling pressure $p_{c r}$. It should be noted that the above equation is independent of the mode number $n$. This shows that only axisymmetric modes can occur in this problem in spite of the inclusion of the Winkler foundation term.

\section{Analytical results and discussion}

\subsection{Buckling pressure}

Figure 3 shows the variation of the nondimensionalized critical buckling pressure $p_{c r} / p_{0}$, where $p_{0}$ is the critical pressure for the empty complete spherical shell that is given by:

$$
p_{0}=\frac{2 E}{\sqrt{3\left(1-\nu^{2}\right)}}\left(\frac{h}{a}\right)^{2} .
$$

As can be easily seen in this figure, the value of $p_{c r} / p_{0}$ increases with the increasing stiffness ratio $a k_{f} / E$. This fact can also be found in Fig. 4 which illustrates the nondimensional critical pressure curves with $a k_{f} / E$ and $a / h$. The variation of the critical buckling mode number $m_{c r}$ is plotted in Fig. 5. Larger values of $a k_{f} / E$ cause higher buckling modes to be triggered, as in the case of beams on an elastic Winkler foundation ${ }^{15)}$.

Figure 6 shows a phase diagram of the buckling mode number $m$. This figure gives significant information on the various axisymmetric buckling modes depending on the values of $a k_{f} / E$ and and $a / h$. It is 


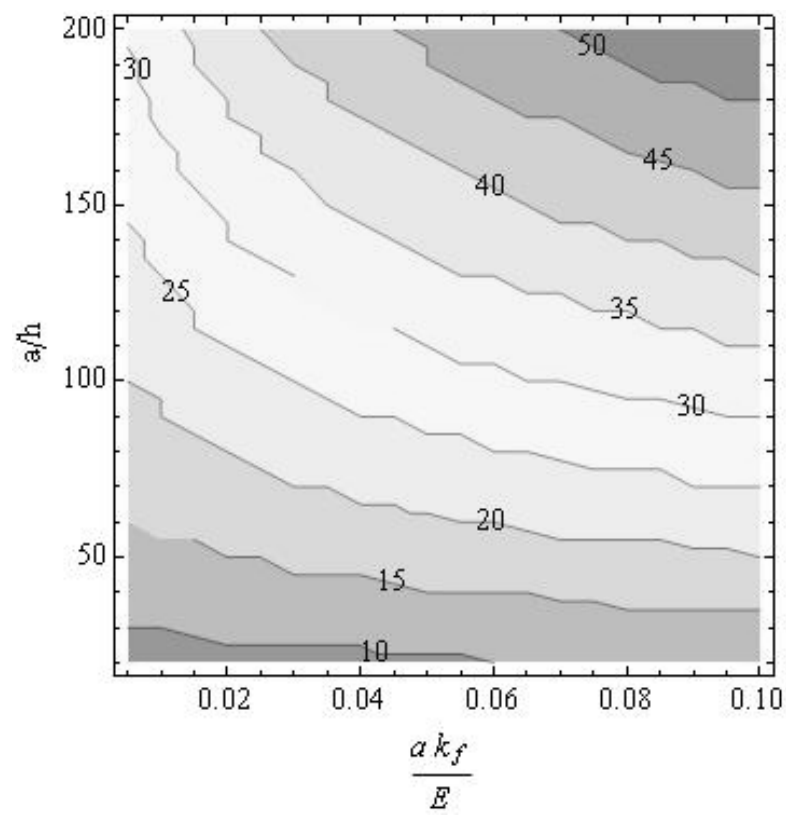

Fig. 6 Phase diagram of the buckling mode number $m$. Various axisymmetric buckling modes can be found depending on the values of $a k_{f} / E$ and and $a / h$.

observed from this figure that larger $a k_{f} / E$ and $a / h$ values favour buckling modes with higher mode numbers. We also find that when the spherical shells are relatively thin with larger $a / h$ values, the effect of the foundation modulus becomes more significant when compared with the case for the relatively thick shells with smaller $a / h$ values.

\subsection{Eigenmodes}

Figure 7 illustrates the buckling eigenmodes for the empty complete spherical shell of $a / h=100$. The characteristic wavy-shaped axisymmetric buckling deformation with the mode number $m=18$ is found in this case. The obtained mode number for this shell is the same as that determined by Koiter ${ }^{6}$.

On the other hand, Fig. 8 shows the eigenmodes for the "filled" spherical shell with $a / h=100$ and $a k_{f} / E=0.1$; the axisymmetric buckling deformation of the mode number $m=33$ is seen in that figure. As can be understood in the developed formulation, non-axisymmetric modes cannot occur under the condition of a constant foundation modulus. However, the cross-sectional shape for this case is moderately different from that shown in Fig. 7.

The results shown in Fig. 7 and 8 suggest that the eigenmodes change corresponding to the mode number $m$. When $m$ is even, the corresponding deformation of the spherical shell is symmetric about the equator, as shown in Fig. 7. On the other hand, when $m$ is odd, the deformation is anti-symmetric about the equator, that is, an inward deformation at the north pole is accompanied by an outward deformation at
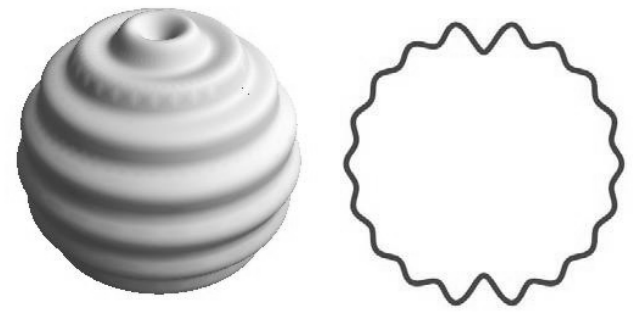

Fig. 7 Buckling modes of an hydrostatically pressurized "empty" complete spherical shell with $a / h=100$ and $a k_{f} / E=0 . \quad$ (Left) $\mathrm{A}$ 3-dimensional view through a great circle. (Right) Cross-sectional view. The buckling mode with mode number $m=18$ is found.
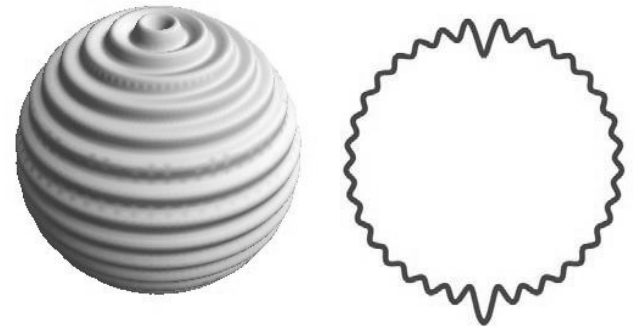

Fig. 8 Buckling modes of an hydrostatically pressurized "filled" complete spherical shell with $a / h=100$ and $a k_{f} / E=0.1$. (Left) $\mathrm{A}$ 3-dimensional view through a great circle. (Right) Cross-sectional view. The buckling mode with mode number $m=33$ is found.

the south pole. This is owing to the property of the geometry of the sphere and the Legendre functions, not whether the shell is empty or otherwise.

\section{Conclusions}

The characteristic buckling modes in hydrostatically pressurized complete spherical shells filled with an elastic medium have been demonstrated. The governing equations of equilibrium have been derived from a theoretical formulation based on smalldisplacement thin shell theory; these have been solved using an exact methodology. From the formulation developed currently it has been shown that only axisymmetric eigenmodes can occur in spite of adding the Winkler foundation term; critical modes that are symmetric or anti-symmetric about the equator may be determined depending on the combination of the foundation stiffness and the radius to wall-thickness 
ratio. A phase diagram has been established to obtain the requisite values of $a / h$ and $a k_{f} / E$ for a corresponding buckling mode.

This work is a fundamental investigation that has been developed with future studies in mind. These will address the post-buckling behaviour ${ }^{16), 17)}$ and non-axisymmetric modes which may occur when a foundation modulus is not a constant value or a function of the wave number of the shell deformation.

\section{Acknowledgements}

We acknowledge the contribution of Professors Takashi Mikami and Shunji Kanie of Hokkaido University for stimulating discussions and helpful comments. This study was conducted during the 6 months that the leading author (MS) spent within the Structures Section of the Department of Civil and Environmental Engineering at Imperial College London as a Visiting Research Fellow. Financial support from Faculty of Engineering, Hokkaido University for the stay is greatly acknowledged. This study was also supported by a Grant-in-Aid for Scientific Research from MEXT, Japan.

\section{REFERENCES}

1) Tarnai, T. Buckling patterns of shells and spherical honeycomb structures. Comput. Math. Appl., Vol.17, pp.639-652, 1989.

2) Baowan, D., Thamwattana, N. and Hill, J. M. Continuum modeling of spherical and spheroidal carbon onion. Eur. Phys. J. D., Vol.44, pp.117123, 2007.

3) Ru, C. Q. Buckling of empty spherical viruses under external pressure. J. Appl. Phys., Vol.105, pp.124701_1-124701_3, 2009.

4) Thompson, J. M. T. The elastic instability of a complete spherical shell. Aero. Quart., Vol.13, pp.189-201, 1962.

5) Hutchinson, J. W.: Imperfection-sensitivity of externally pressurized spherical shells. J. Appl. Mech., Vol.34, pp.49-55, 1967.
6) Koiter, W. T.: The nonlinear buckling problem of a complete spherical shell under uniform external pressure. Proc. R. Dutch Acad. Sci. B, Vol.72, pp.40-123, 1969.

7) Yin, J., Cao, Z., Li, C., Sheinman, I. and Chen, $\mathrm{X}$. Stress-driven buckling patterns in spheroidal core/shell structures. Proc. Natl. Acad. Sci. U.S.A., Vol.105, pp.19132-19135, 2008.

8) Timoshenko, S. P. and Gere, J. Theory of elastic stability. McGraw-Hill, 1973.

9) Flügge, W. Stresses in shells. Springer-Verlag, Heidelberg 1973.

10) Fok, S. L and Allwright, D. J. Buckling of a spherical shell embedded in an elastic medium loaded by a far-field hydrostatic pressure. J. Strain Anal. Vol.36, pp.535-544, 2001.

11) Shima, H., Sato, M., Iiboshi, K., Ghosh, S. and Arroyo, M. Diverse corrugation pattern in radially shrinking carbon nanotubes. Phys. Rev. B, Vol.82, pp.085401_1-085401_7, 2010.

12) Sato, M., Shima, H. and Iiboshi, K. Core-Tube morphology of multiwall carbon nanotubes. Int. J. Mod. Phys. B, Vol.24, pp.288-294, 2010.

13) Brush, D.O. and Almroth, B.O. Buckling of Bars, Plates, and Shells, McGraw-Hill, New York 1975.

14) De Souza, V. C. M. and Croll, J. G. A. An energy analysis of the free vibrations of isotropic spherical shells, J. Sound Vibr., Vol.73, pp.379-404, 1980.

15) Wadee, M. K., Hunt, G. W. and Whiting, A. I. M. Asymptotic and Rayleigh-Ritz routes to localized buckling solutions in an elastic instability problem, Proc. R. Soc. A, Vol.453, pp.2085-2107, 1997.

16) Thompson, J. M. T. and Hunt, G. W. A general theory of elastic stability, John Wiley \& Sons, London 1973.

17) Thompson, J. M. T. and Hunt, G. W. Elastic instability phenomena, John Wiley \& Sons, Chichester 1984 .

(Received March 8, 2011) 